\title{
1 Unlocking the functional potential of polyploid yeasts
}

2 Simone Mozzachiodi ${ }^{1,2}$, Kristoffer Krogerus ${ }^{3}$, Brian Gibson ${ }^{3, \#}$, Alain Nicolas ${ }^{1,2,4}$ and Gianni Liti $^{1}$

4 Affiliations

5 'Université Côte d'Azur, CNRS, INSERM, IRCAN, Nice, France.

$6 \quad{ }^{2}$ Meiogenix, 38, rue Servan, Paris 75011, France.

$7 \quad{ }^{3}$ VTT Technical Research Centre of Finland Ltd., Espoo, Finland.

8 Institut Curie, Centre de Recherche, CNRS-UMR3244, PSL Research University, Paris 75005,

9 France.

\section{Notes}

12 \#Current address: Institute for Food Technology and Food Chemistry Department of Brewing and

13 Beverage Technology, Technical University, 13353, Berlin, Germany

14

15 Correspondence and requests for materials should be addressed to:

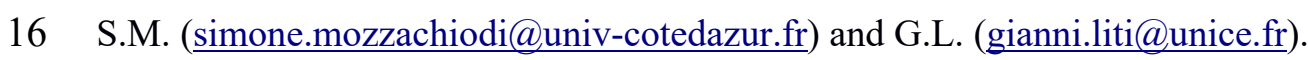




\section{7}

Breeding and domestication have generated widely exploited crops, animals and microbes. However, many Saccharomyces cerevisiae industrial strains have complex polyploid genomes and are sterile, preventing genetic improvement strategies based on breeding. Here, we present a novel strain improvement approach based on the budding yeasts' property to promote genetic recombination when meiosis is interrupted and cells return-to-mitotic-growth (RTG). We demonstrated that two unrelated sterile industrial strains with complex triploid and tetraploid genomes were RTG-competent and developed a visual screening for easy and highthroughput identification of recombined RTG clones based on colony phenotypes. Sequencing of the evolved clones revealed unprecedented levels of RTG-induced genome-wide recombination. We generated and extensively phenotyped a RTG library and identified clones with superior biotechnological traits. Thus, we propose the RTG-framework as a fully non-GMO workflow to rapidly improve industrial yeasts that can be easily brought to the market.

\section{Introduction}

Humans have selected and improved organisms for centuries, leaving profound hallmarks of domestication in their genomes and lifestyles ${ }^{1}$. Selective breeding, in which hybrids with improved performance are generated, was one of humanity's first biotechnology advances and it is still widely applied ${ }^{2}$. The advent of new genetic engineering techniques enabled to directly manipulate core biological traits relevant for human activities ${ }^{3}$. However, the exploitation of genetically modified organisms (GMOs) in the food sector remains controversial, highly regulated and restricted in many countries.

Humans unwittingly domesticated yeasts of the genus Saccharomyces since the earliest food and beverages fermentations were carried out ${ }^{4-6}$. Since then, novel fermentation processes selected new yeast strains that are still used nowadays ${ }^{7}$. Nevertheless, there is a pressing interest across different sectors that exploit Saccharomyces yeasts to create new strain variants able to better tolerate stresses encountered during the industrial fermentations or increase the yield of desired compounds. Newly designed de novo lab-hybrids that combine different Saccharomyces species have displayed good or superior fermenting properties compared to common commercial strains suggesting that indeed commercial strains are not yet optimized, leaving room for improvement despite the long domestication period ${ }^{8-10}$. However, attempts to cross industrial yeasts through selective breeding or induce genomic recombination through meiosis are often unsuccessful due to the inherent sterility of the strains, which is a hallmark of yeast domestication ${ }^{6,11}$. This domestication-syndrome might have derived from the lack of selection on sexual reproduction, random genetic drift or adaptation to specific fermentation niches, leading to the accumulation of punctuated deleterious loss-of-functions (LOF) alleles that inactivate genes involved in the gametogenesis (sporulation in yeast). In addition, the genomes of industrial strains often have features such as extreme sequence divergence between the subgenomes (namely 


\section{Results}

\section{Genomic and reproductive portraits of industrial polyploid yeasts} directly fixing the lack of a complete sexual reproduction remains unfeasible. traits.

heterozygosity), structural rearrangements, aneuploidy and polyploidy all of which are known to contribute to sterility $^{12}$. Classic examples are strains used in beer production or in the baking industry sharing different ancestries that have repeatedly converged toward polyploid complex genomes ${ }^{5,13,14}$. However, tetraploid strains derived from designed crosses perform a correct chromosome segregation and produce viable gametes ${ }^{15}$ in contrast to triploid strains. Therefore, the genetic basis driving this extreme sterility are not yet fully understood and multiple factors likely contribute to impair the sexual reproduction of industrial polyploid strains. Thus, other approaches to improve sterile industrial strains have been proposed ${ }^{16}$ because

Recently, we demonstrated that aborting meiosis in laboratory-derived sterile diploid hybrids and returning them to mitotic growth, a process called return-to-growth (RTG), generated genetic diversity and resulted in phenotypic variability in the evolved samples ${ }^{17,18}$. RTG is induced when cells that have entered meiosis but are not yet committed to complete it are shifted back to a nutrient-rich environment ${ }^{19}$. As in normal meiosis, after DNA replication Spo11p induces multiple genome-wide double-stranded breaks (DSBs) that lead to the formation of intermediate recombinant molecules ${ }^{20}$. These molecules are then resolved upon the resumption of the mitotic cycle, resulting in dispersed $\mathrm{LOH}$ tracts that derive from the segregation of the chromatids in the mother and the daughter cells. Despite generating multiple dispersed LOH tracts, the RTG process is not mutagenic and it preserves the initial genome content in diploid laboratory strains ${ }^{17}$. Furthermore, the LOHs induced by RTG can lead to rapid phenotypic diversification through the unmasking of beneficial alleles ${ }^{17,18}$. Given that RTG induces phenotypic variation without complete sexual reproduction, which often is defective in industrial strains, RTG may represent a powerful approach for improvement of industrial strains. However, it is still unknown whether the RTG paradigm can induce recombination and unlocks novel phenotypic variability without triggering systemic genomic instability in such complex genomic scenarios. Furthermore, the selection of RTG recombinant clone either requires genetically-engineered selective markers ${ }^{17}$ or a lengthy and low throughput microdissection approach ${ }^{18}$. This hinders the scaling-up of the RTG process to generate a large library of recombined industrial yeasts. Here, we developed a comprehensive workflow to improve polyploid industrial yeasts through RTG and easily select RTG-recombined clones. We applied this workflow to generate a non-GMO library of evolved industrial polyploid strains harbouring improved biotechnological

Domesticated strains derived from wild yeast ancestors show hallmarks of genomic complexity such as polyploidy, aneuploidy and horizontal gene transfer (HGT) (Fig. 1a). In order to develop a tailored RTG framework for industrial strains (Fig. 1b), we selected two genetically unrelated industrial S. cerevisiae strains as test cases, hereafter called OS1364 and OS1431 (Fig. 1a), and characterise their genomes and reproductive capacity. OS1364 was isolated from a cassava factory in Brazil and belongs to the mosaic beer clade, whereas OS1431 was isolated from an ale beer fermentation in England and belongs to the ale beer clade ${ }^{13}$ 
(Supplementary table 1). We performed both short- and long-reads sequencing and detected multiple hallmarks of domestication. Despite their genetic ancestry, both strains are polyploid, with OS1364 being triploid (3n, with one additional copy of chromosome III) and OS1431 being tetraploid (4n) (Supplementary Figure 1a-b). Furthermore, both genomes harbour a considerable number of heterozygous positions ( $40 \mathrm{~K})$ distributed genome-wide suggesting that these two strains are intraspecies hybrids generated by admixture of different S. cerevisiae strains (Fig. 1c, Supplementary Figure 1c). A large region of loss-of-heterozygosity (LOH) is present on chromosome XII downstream of the rDNA locus in both strains (Supplementary Figure 1c), consistent with this locus being inherently prone to recombination ${ }^{5,13}$. We detected several singlenucleotide polymorphisms within coding regions ( $n=28932$ OS1364, $n=32399$ OS1431) with OS1431 harbouring more than the expected number (Expected $\sim 75 \%$, based on the reference genome, Observed $82.2 \%$ ). Among these polymorphisms we found pervasive heterozygous missense variants ( $n=10747$ OS1364, $n=13033$ OS1431, Supplementary Figure 1d). We observed that the genetic variation also manifests in the form of copy number variants (CNVs) (Supplementary Figure 1b), resulting in small amplifications or deletions similar to those previously observed in industrial strains ${ }^{5}$. Subtelomeric regions are known to be highly enriched in this type of variation ${ }^{21}$. For instance, we detected a large horizontal gene transfer (HGT) region close to the subtelomere XIII-R of OS1364 (present in two homologs) (Supplementary Figure 1e). HGT regions are known to harbour genes that improve fitness in the fermentation environment and have been detected often in domestic strains ${ }^{22}$. The genetic diversity observed in the two strains might reflect the different evolutionary histories of the haplotypes before or after the admixture.

Next, we investigated the reproductive capacity of these polyploid strains and observed a defective sexual reproduction (Fig. 1d). Specifically, the strains showed slow, asynchronous and inefficient meiotic progression and generated nearly completely unviable gametes (less than 1\%). The high heterozygosity and lack of an effective sexual reproduction suggest that the genetic load might contribute to gametes' unviability. By using Ensamble Variant Effect Predictor (VEP) (Methods), we detected many highly deleterious variants in both genomes (start-loss, stop-loss, stop-gain, $n=207$ OS1364, $n=338$ OS1431), including 10 (OS1364) and 24 (OS1431) in essential genes ${ }^{23}$ (Fig. 1e, Supplementary Tables 2 and 3). OS1364 has accumulated a number of deleterious variants not significantly different from the average number of LOFs observed in domesticated strains of the 1011 S. cerevisiae collection ${ }^{11}$, whereas OS1431 has accumulated significantly more LOFs (Supplementary Figure 1f). Given OS1431 is tetraploid and tetraploid strains correctly segregate their chromosomes in contrast with triploid strains ${ }^{24}$, the observed genetic load is likely to drive part of its sterility. However, we performed a GO-term analysis to test if genes involved in the sporulation process had accumulated LOFs and we did not find any enrichment of LOF in sporulation associated genes in frame with the results that cells of both strains entered meiosis when starved. Overall, these two sterile domesticated $S$. cerevisiae strains with complex polyploid genomes represent ideal test cases to probe the RTG framework. 


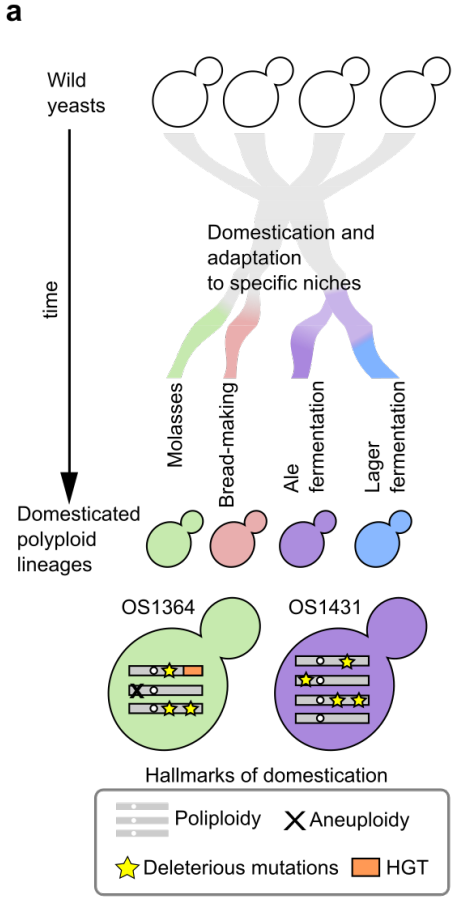

b

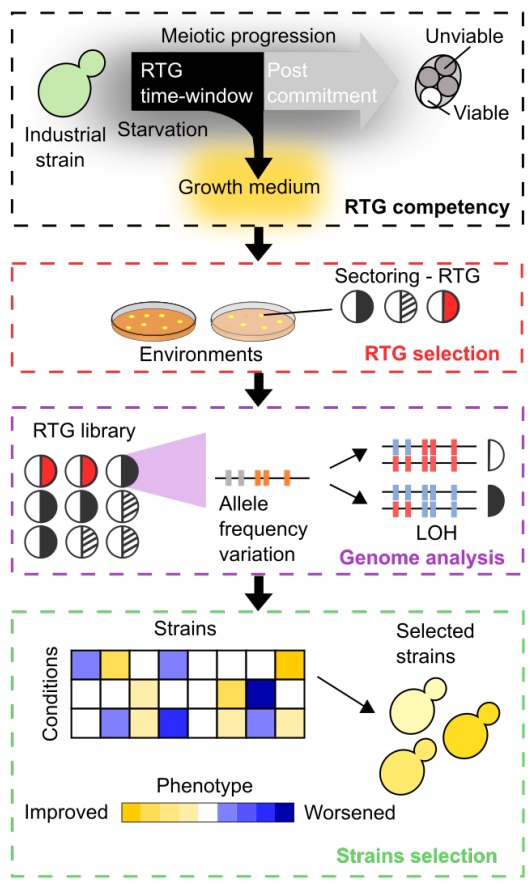

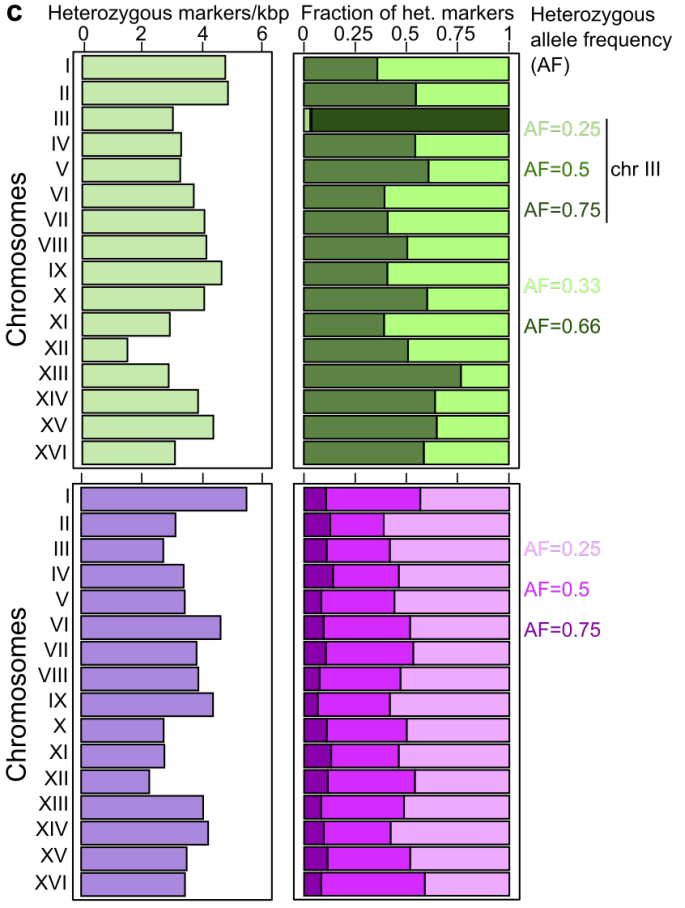

d
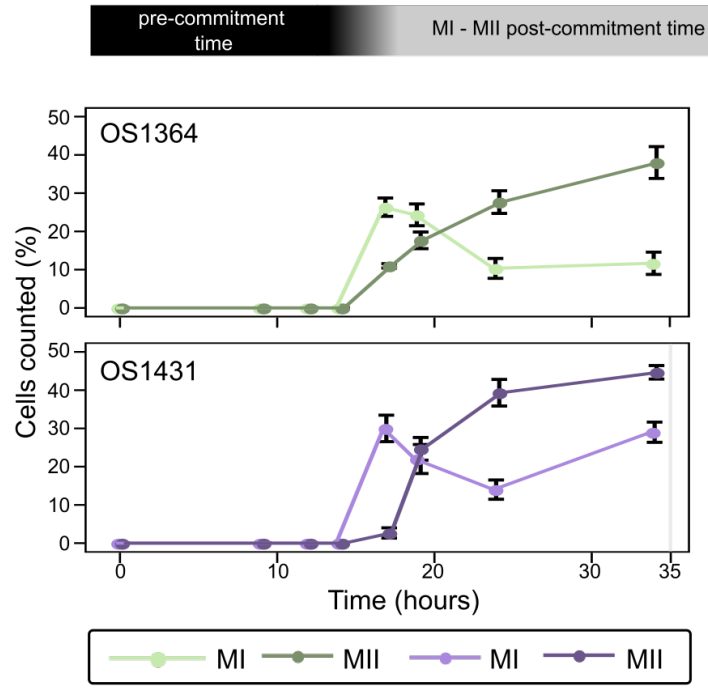

Fig. 1. Genome complexity and sterility of domesticated $\boldsymbol{S}$. cerevisiae | (a) Domestication produced polyploid strains adapted to specific human-made niches that are characterised by common genomic hallmarks (bottom box), which are detected in both strains used in this study. (b) Schematic depicting the RTG framework: we initially quantify and optimise the meiotic progression, followed by selection of novel colony phenotypes. Finally, RTG samples are sequenced and phenotyped to identify clones with improved industrial traits. (c) Left: Level of heterozygosity (number of heterozygous markers/kbp) across each chromosome, total number of heterozygous markers ( $n=40431$ OS1364 (green), $n=39376$ OS1431 (purple)). Right: Heterozygous markers partitioned according to their allele frequency. (d) Meiotic progression measured by nuclei DAPI staining, reported as the average ( $n=3$ replicates) of the percentage of cells that have progressed over the first (MI) and second (MII) meiotic division. The error bars represent the standard deviation. On the right, bar plot representing the spore viability (3 viable spores/400 OS1364, 1 viable spore/400 OS1431). The top arrow is a qualitative description of the meiotic progression based on the results of the DAPI staining. (e) The bar plot represents the number of high-impact variants affecting essential (red) or non-essential (grey) genes and further divided into homozygous (Hom) if present in all the haplotypes, and heterozygous (Het) if not present in all the haplotypes. 


\section{Sterile polyploids are RTG competent}

140 Despite the fact these polyploid $S$. cerevisiae hybrids show an inefficient meiotic progression and generate 141 inviable gametes, we conjectured that RTG should not be precluded since it only relies on the meiotic 142 prophase progression, a time window in which cells are not yet committed to complete meiosis. To test if our 143 two polyploid hybrids are able to perform RTG, we engineered them with a genetic system to measure $\mathrm{LOH}$ 144 rates at a heteroallelic LYS2/URA3 locus on chromosome II, that we have broadly applied in RTG 145 experiments ${ }^{17,25}$ (Fig. 2a). We validated the genotype of the engineered LYS2/URA3 strains by PCR and 146 growth on selective media (Supplementary Figure 2a, Supplementary Table 4). Then, we evolved the 147 engineered OS1364 ${ }^{L Y S 2 / U R A 3}$ and OS1431 $1^{\text {LSS2/URA3 }}$ through RTG and collected cell populations throughout the 148 meiotic progression (Fig. 2b). We calculated the RTG-induced recombination by comparing the basal level of 149 cells growing on 5-FOA measured in the unsporulated cultures (T0) to the RTG cells obtained after 6 hours 150 (T6) and 14 hours (T14) of sporulation induction (Methods). We detected a 10-fold and 3-fold increase of 151 cells growing on 5-FOA, indicating an increased LOH rate at T14 in OS1364 ${ }^{\text {LSS2/URA3 }}$ and in OS1431 $1^{\text {LSS2/URA3 }}$ 152 respectively, whereas the increase was not significant at $\mathrm{T} 6$, consistent with the fact that cells have not 153 progressed sufficiently through meiosis at this timepoint (Fig. 2b, Supplementary Table 5). The absolute 154 percentage of cells grown at T14, calculated subtracting the T0 background value to the T14 (Methods), was 155 almost 2-fold higher in OS1431 $1^{\text {LSS2/URA3 }}\left(0.10 \%\right.$ in OS1364 ${ }^{\text {LSS2/URA3 }}, 0.19 \%$ OS1431 $\left.1^{\text {LS2/URA3 }}\right)$, in line with its 156 lower heterozygosity on chromosome II (Fig. 1c) where pre-existing small LOH regions might represent 157 preferential sites of recombination ${ }^{25}$. To further prove that RTG induced the increase in recombination 158 observed at the LYS2/URA3 locus we deleted all three copies of SPO11, which is essential for inducing DSBs 159 in meiosis, in OS1364 ${ }^{\text {LYS2/URA3 }}$ (Supplementary Figure 2b) and measured recombination. We did not detect 160 any significant increase between the T0 and the respective T14 in the OS1364 ${ }^{\text {LSS /URA3 }}$ spol14 strain 161 (Supplementary Figure 2c, Supplementary Table 5), supporting the conjecture that RTG caused the 162 increased recombination as it relies on the Spo11p induced DSBs in early meiosis.

163 We next performed whole-genome sequencing of the LYS2/URA3 parental strains, controls (T0) ( $n=2$ 164 OS1364, $n=2$ OS1431, Table S1) and RTG-evolved clones (T14) ( $n=11$ OS1364, $n=11$ OS1431, Table S1) 165 isolated on 5-FOA plates to evaluate the genome-wide impact of RTG. Our analyses revealed varying levels 166 of recombination in the RTG clones, consistent with previous reports in RTG diploid hybrids ${ }^{17,18}$ (Fig. 2c-d, 167 Supplementary Figure 2d). The RTG clones derived from OS1364 ${ }^{L Y S 2 / U R A 3}$ had an average of 10\% (maximal 168 value $26 \%$ ) of the genome affected by $\mathrm{LOH}$, whereas OS1431 $1^{\text {LYS2/URA3 }}$ had an average of $6.7 \%$ (maximal value 169 13.5\%). Moreover, we did not detect any chromosome loss potentially accounting for 5-FOA resistance in the 170 RTG sequenced clones, showing that despite the strain polyploidy, LOH at the LYS2/URA3 locus arose more 171 frequently than aneuploidy during RTG. We observed overall genome-wide stability and detected only one 172 aneuploidy and two large CNVs across the 11 RTGs derived from OS1431 $1^{\text {LSS2/URA3 }}$, where the two CNVs 173 likely resulted from ectopic recombination initiated by a small region of homology between chromosome IX 174 and chromosome XIV (Supplementary Figure 3a-b-c). Similarly, we detected only two aneuploidies in one 175 RTG sample across the 11 samples derived from OS1364 ${ }^{L Y S 2 / U R A 3}$. Altogether, these results demonstrate that 
a

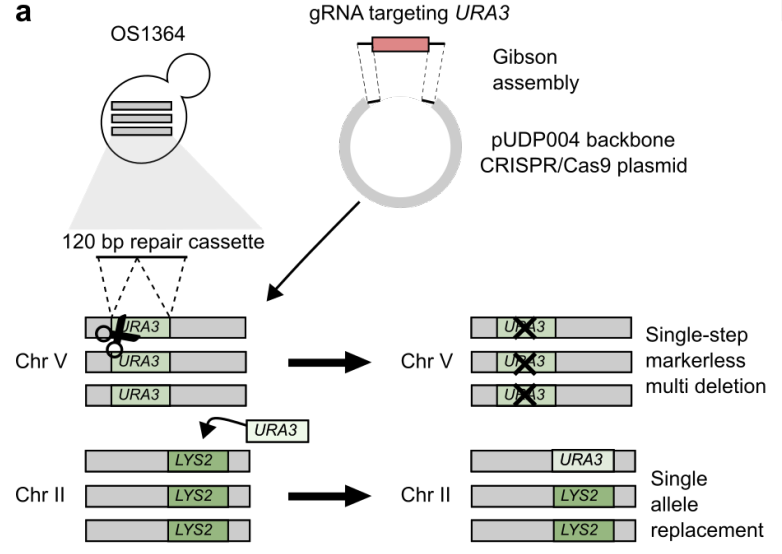

c

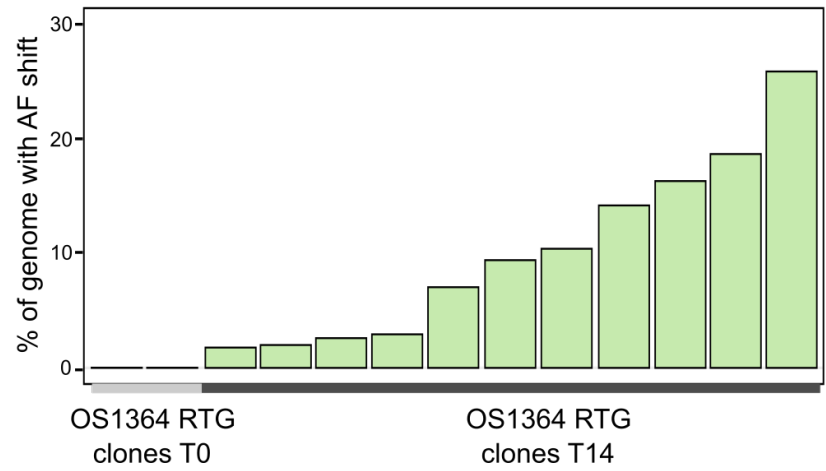

b

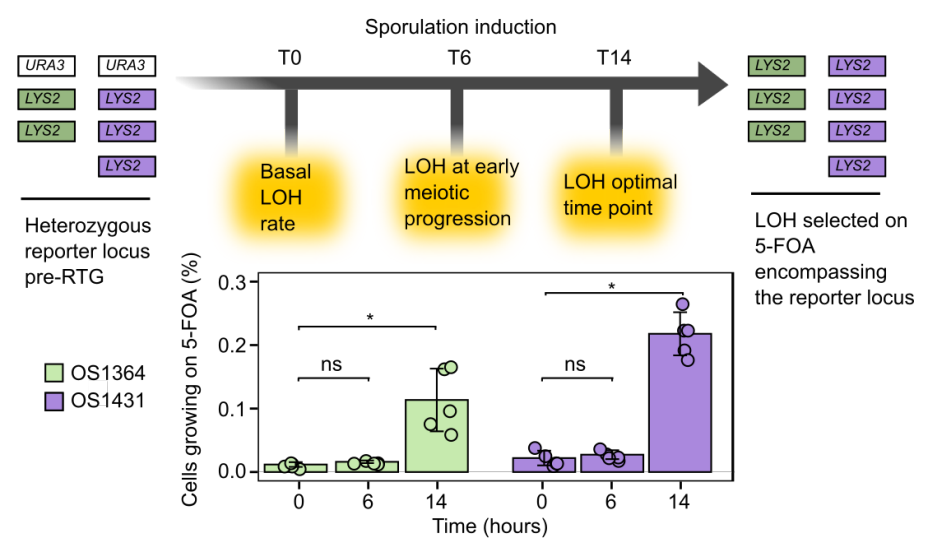

d

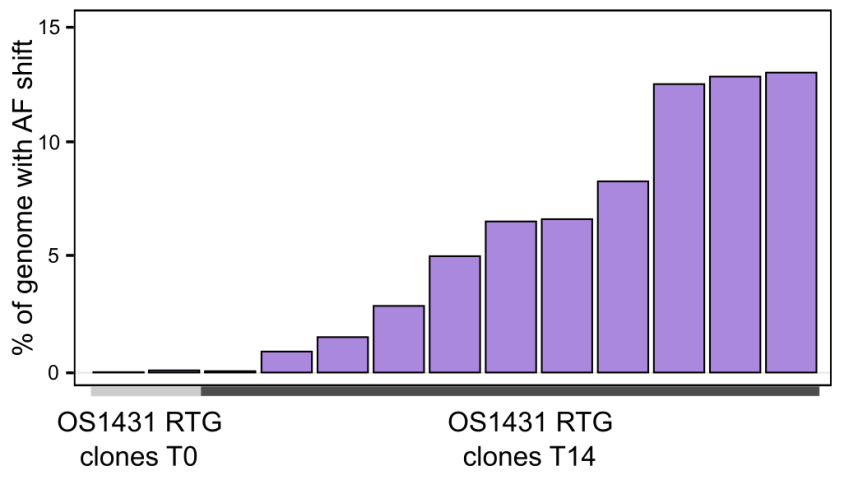

Fig. 2. RTG induced recombination in polyploid sterile hybrids | (a) A CRISPR-based approach enabled the engineering of the URA3-loss genetic system in the polyploid strains. The strains generated in such way are hereafter referred to as LYS2/URA3 regardless of the number of loci. (b) Reporter assay used to measure recombination rate at the heterozygotic locus LYS2/URA3 (top panel). Bar-plots representing the average ( $n=5$ replicates) percentage of cells growing on 5-FOA at different time points: no sporulation induction (T0), 6 hours (T6) and 14 hours (T14) of sporulation induction (bottom panel). The increase of cells growing on 5-FOA at T14 compared to T0 was significant for both samples ( $p$-value $<0.05$, one-sided Wilcoxon ranked-sum test) whereas was not significant at T6 ( $p$-value $>0.05$, onesided Wilcoxon ranked-sum test). The error-bars represent the standard deviation. (c-d) Bar plot representing the percentage of genome in which we detected an allele frequency (AF) shift for the RTG samples derived from OS1364 LYS2/URA3 (green, c) and OS1431 $1^{\text {LYS2/URA3 }}$ (purple, d). Recombination and LOH induced upon genome engineering with CRISPR/Cas9 at the native URA3 locus was excluded.

\section{Selection of recombined RTG clones by natural colony phenotypes}

196 The RTG selection based on URA3-loss requires genome editing and produces GMOs that have marketing 197 constraints in the food industry. To overcome this limitation, we devised an alternative selection strategy based on natural variability in colony phenotypes such as morphology and colour. Colony phenotypes are complex traits, influenced by both genetic and environmental factors (Fig. 3a). We used a YPD-based medium with varying concentrations of dextrose $(0.5 \%$ and $1 \%)$, a major environmental regulator of colony 
morphology ${ }^{26,27}$, to unveil novel phenotype of colonies derived from RTGs of OS1364 and OS1431 wild-type (WT) cells (Fig. 3a, Methods). Indeed, we found phenotypic variability in the RTG colonies but the divergent phenotype was often present in only a large sector (approximately half) of the colony. Therefore, we hypothesized that the sectored colonies were mother-daughter (M-D) RTG pairs that did not complete budding at the time of plating. Consistently, we found sectored and non-sectored colonies according to when cells were plated after the RTG induction (Supplementary Figure 4a). We observed three types of sectored colony phenotypes in the RTG plates of OS1364 (wrinkled-smooth, red-white and dark-white) (Fig. 3b,

Supplementary Figure 4, Supplementary Table 5) with a summed total frequency of $\sim 0.75 \%$ and one type of sectored colony in the OS1431 RTGs (wrinkled-smooth) with a frequency of $\sim 0.5 \%$ across all environments tested (Fig. 3b, Supplementary Table 5). In contrast, we did not observe any of these phenotypes in control plates where cells were plated without inducing sporulation. Thus, this result was in line with our initial hypothesis that the sectored colonies were unveiled in the modified YPD plates upon budding of recombined mother-daughter (M-D) RTGs, whereas the non-sectored colonies displaying phenotype variation represent RTG cells in which the mother and daughter were separated by the first budding before the plating. An alternative explanation is that the sectored RTG colonies derived from residual sporulation and spore germination, although this is unlikely given the near to zero spore viability observed. To undoubtedly exclude this scenario, we deleted the NDT80 gene by CRISPR/Cas9 multi-deletion in both hybrids, to generate mutants that are RTG competent ${ }^{17,20}$ but arrest before the first meiotic division (MI) and therefore unable to complete sporulation (Supplementary Figure 5a-b). Then, we evolved the OS1364 ndt804 and OS1431 $1^{\text {ndt } 804}$ mutants through the same RTG protocol used for the wild-type hybrids (Supplementary Figure

5b) and detected sectored phenotypes similar to those seen in the WT hybrids, thus excluding the possibility that residual sporulation contributes to the formation of sectored colonies.

We sequenced multiple paired samples derived from sectored RTG colonies and non-sectored RTG colonies derived from RTG in the OS1364 and OS1341wild-type and ndt804 strains and samples isolated from control plates (T0) (Supplementary Table 1). Whole-genome sequencing revealed recombination detected as allele frequency (AF) shifts across the unphased heterozygous markers in all the putative M-D RTG colonies with a sectored phenotype (Fig. 3c-d, Supplementary Figure 5c-d). The fraction of the genome in which we detected RTG-induced recombination was highly variable in both strains $(9.7 \pm 8.2 \%$ in OS1364, $4.8 \pm 2.4 \%$ in OS1431 WT-RTG, average $\pm \mathrm{SD}$ ), mirroring the results obtained from the URA3-loss assay. Moreover, we provided two additional proofs that the sectored RTG colonies were genuine RTG M-D pairs. First, the AF shift for heterozygous markers was largely reciprocal in the wild-type and $n d t 80 \Delta$ sectored RTG pairs with the rest of the non-reciprocal events representing gene-conversion (Fig. 3e, Supplementary Figure 6a-b-c, Supplementary Table 12) as found in M-D RTG pairs of diploid lab strains. In addition, by comparing each pair-wise combination of RTG M-D pairs we also found that each pair is clearly differentiated from the others in term of recombination landscape (Supplementary Figure 6d). 
238 were driven by localised LOH. Second, we observed a complementary gain and loss of one chromosome in 239 one M-D pair derived from OS1364 (Supplementary Figure 7b), which is compatible with chromosome 240 missegregation between M-D during RTG. Thus, we concluded that the sectored RTG colonies were indeed 241 M-D pairs and we exploited the recombination generated by RTG in OS1364 M-D RTGs to produce phased 242 local haplotypes showing a potential application of RTG in polyploid yeasts (Supplementary Figure 7c).

243 Overall, we showed that highly recombined RTG M-D pairs can be selected by exploiting natural colony 244 phenotypic variation and we proved that these phenotypes arise as a result of RTG-induced recombination. 
bioRxiv preprint doi: https://doi.org/10.1101/2021.10.21.465299; this version posted October 22, 2021. The copyright holder for this preprint (which was not certified by peer review) is the author/funder, who has granted bioRxiv a license to display the preprint in perpetuity. It is made available under aCC-BY-NC-ND 4.0 International license.
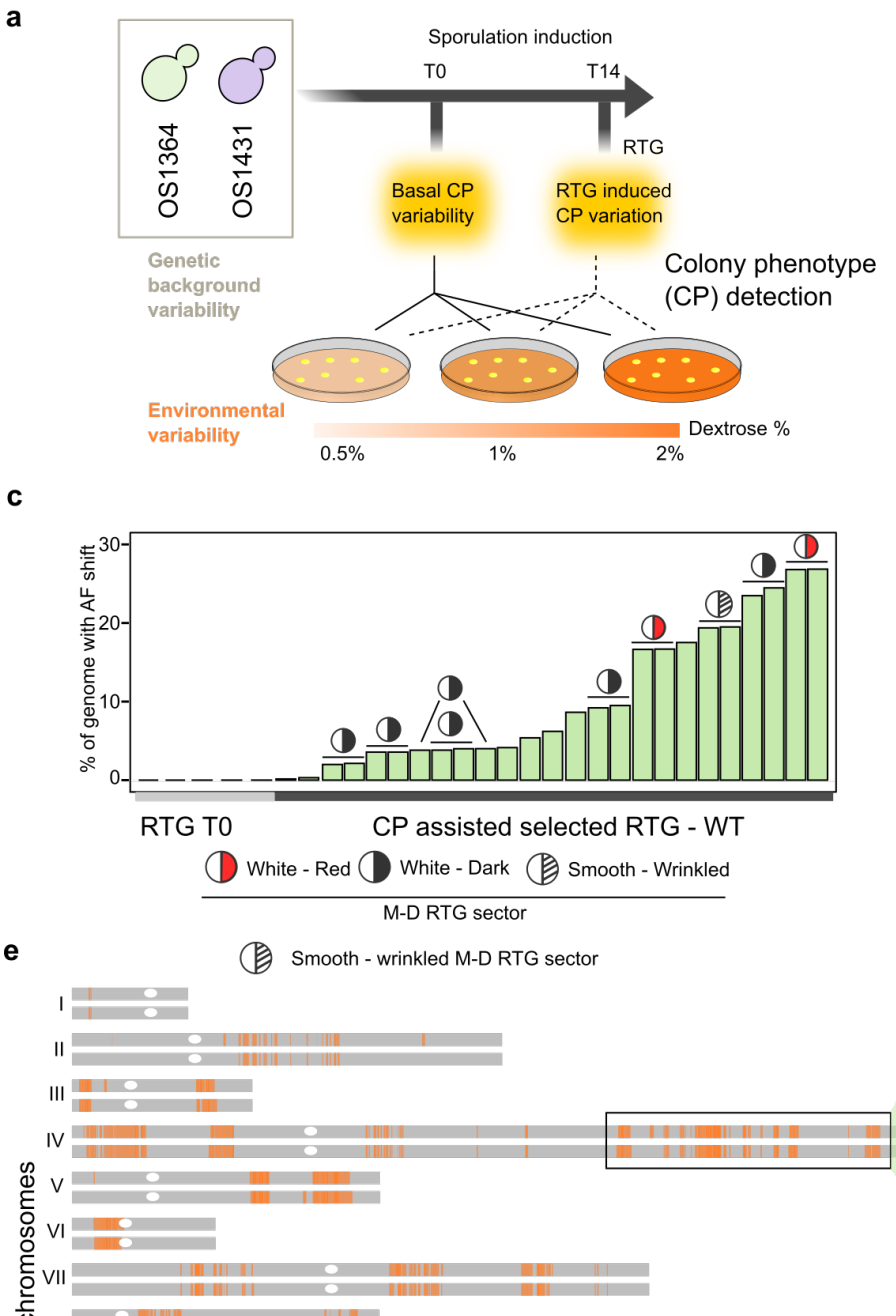

b
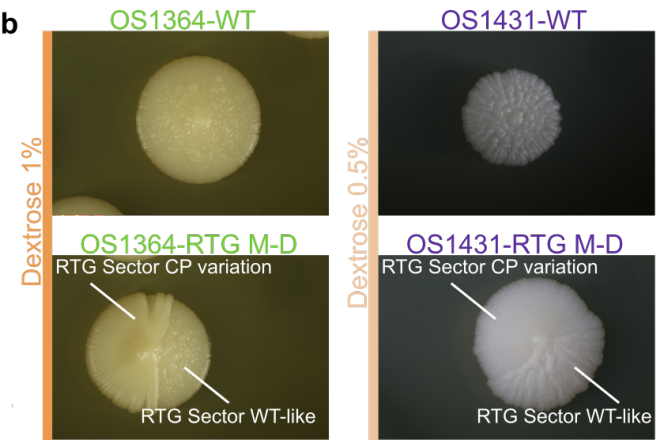

d

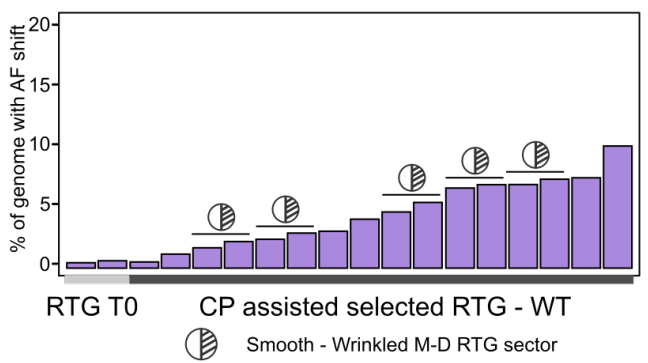

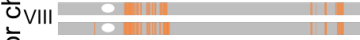

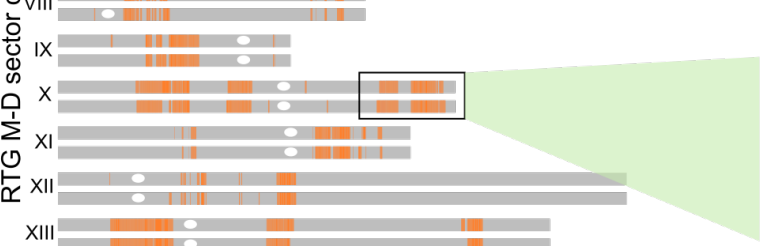

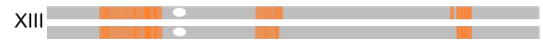

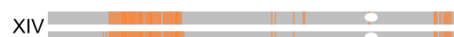

$\mathrm{XV}$

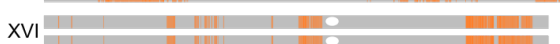

No allele frequency shift detected

Allele frequency shift detected

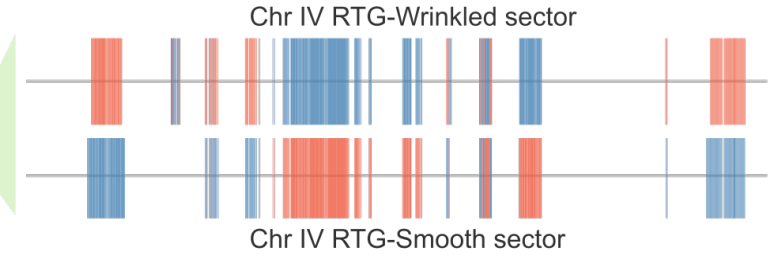

Chr X RTG-Wrinkled sector

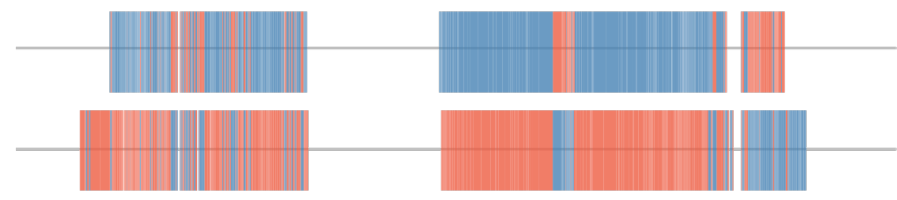

Chr X RTG-Smooth sector

Gain one copy of reference allele Ref/Ref/Alt $\rightarrow$ Ref/Ref/Ref Ref/Alt/Alt $\rightarrow$ Ref/Ref/Alt
Gain one copy of alternative allele Ref/Ref/Alt $\rightarrow$ Ref/Alt/Alt Ref/Alt/Alt $\rightarrow$ Alt/Alt/Alt

Fig. 3. Colony phenotype variability revealed mother-daughter RTG pairs | (a) Varying dextrose concentrations unveiled hidden colony phenotypic variation upon RTG. (b) Wild-type (WT) colony phenotypes (top) and sectored phenotypes emerging on RTG plates (bottom). The concentration of dextrose in the media is reported on the left. (c) The percentage of markers with allele frequency (AF) shift in the OS1364 derived RTG samples. The mother-daughter pairs for which both sectors were sequenced are indicated (circles). Single samples represent non-sectored RTG colonies $(n=4)$ or only one-side of a RTG sectored colony $(n=3)$. (d) As in panel $\mathbf{c}$ for the OS1431 background. (e) Recombination map of a RTG M-D pair (top chromosome: sm244/ bottom chromosome: sm245, derived from OS1364). Grey regions indicate the heterozygous markers without $\mathrm{AF}$ shifts, whereas orange regions contain heterozygous markers with $\mathrm{AF}$ shifts underlying LOH events. A zoom-in of two recombined regions is illustrated on the right. The colour code represents the genotype variation of the heterozygous markers so that the gain of a reference allele given the initial genotype is indicated in blue, whereas the gain of an alternative allele is indicated in red. 


\section{RTG recombination improves industrial fitness}

261 Next, we probed the potential of RTG to improve industrial phenotypes. First, we compared the fermentation 262 performances of OS1364 and OS1431 to the commercial strain WLP001 used as a reference to evaluate the 263 fermentation performances of our strains (Supplementary Table 1). We confirmed that our two selected 264 strains are competitive for industrial fermentation, with OS1364 outperforming both WLP001 and OS1431 for 265 fast fermentation (Supplementary Table 10). However, both OS1364 and OS1431 had a substantial decline in cell viability (of $\sim 25 \%$ and $\sim 50 \%$ respectively) often observed in chronologically aged polyploid $S$. cerevisiae $^{l 1}$, which is more modest in WLP001 (8\%, Supplementary Table 11).

We started our phenotypic screening to identify potential improved RTG variants by evaluating selected nonGMO RTGs ( $n=25$ OS1364, $n=16$ OS1431, Supplementary Table 6) and compared them to their respective parental strain and T0 controls ( $n=2$ OS1364, $n=2$ OS1431) across several conditions mirroring industrial fermentations (Fig. 4a). We performed a first phenotypic screening in osmotic and alcoholic stress conditions and found that RTG samples had broad phenotypic variability with either a worsened, unchanged or improved phenotype as compared to the parental strains and the T0 controls (Fig. 4b, Supplementary Table 6). This scenario is consistent with the LOHs induced by RTG arising randomly in the genome and not as a by-product of a specific selective pressure. Moreover, some M-D RTG pairs showed complementary growth-rate variation (Fig. $4 \mathbf{c}$ ) that can be explained by complementary LOHs segregating weaker and stronger alleles in the RTG pair. Growth rate variation in ethanol and maltose appeared to be moderately correlated in the OS1364 RTG library, underscoring that recombination might have affected pleiotropic genes regulating both traits (Supplementary Figures 8a-b).

Next, we selected RTG samples derived from OS1364 based on their phenotypic performance $(n=12)$ and their LOH landscape across a core set of fermentation genes $(n=74)$, and all the M-D RTG pairs from OS1431 $(n=10)$ (Supplementary Tables 7 and 8). We inoculated the selected RTGs in a high-gravity wort $\left(32^{\circ}\right.$ Plato), representing extreme fermentative conditions, to further investigate differences in stress resistance, carrying out a flask-scale fermentation for 13 days evaluating mass loss and ethanol produced at regular intervals (Supplementary Table 9). Some RTG samples showed increased fermentation kinetics (Fig. 4d) as well as a superior alcohol production (Supplementary Figure 8c) compared to the parental strains. We also noticed that in some M-D RTGs derived from OS1431 the fermentation performances were complementary (Supplementary Figure 8d, Supplementary Table 9). We hypothesized that RTG samples showing improved fermentation performances should also have improved resistance to fermentation stress and, potentially, higher post-fermentation cell viability. Therefore, we selected two RTG variants for each background among the best performers in the previous flask-scale fermentation, to carry on a 2-liter scale fermentation in high-gravity wort, to mimic modern industrial brewery practice $\left(20^{\circ}\right.$ Plato). We found that OS1364 RTGs performed at least equally well as their respective parental strains, while OS1431 RTGs were slightly better in fermentation performances (Supplementary Figure 9a, Supplementary Table 10) but did not have increased post-fermentation viability (Fig. 4e). Remarkably, one OS1364 RTG had a large increase 
296 in post-fermentation cell viability (Fig. 4e, Supplementary Table 11), reaching a level similar to WLP001, 297 showing that RTG can fix detrimental traits.

298 Furthermore, we evaluated the aroma profile and the post-fermentation residual sugars, two parameters that 299 are highly relevant in the beer industry. The two parental strains produced distinct aroma profiles from that of 300 WLP001 and consumed almost all the sugars present in the wort (Supplementary Table 11). The four 301 selected RTG samples did not show any deleterious trade-off in either phenotypes with the exception of a 302 slight increase of acetaldehyde, in sm408, which is not desirable but the increase was negligible (Sensory 303 threshold $=10 \mathrm{mg} / \mathrm{mL}, \mathrm{sm} 408=10.5 \mathrm{mg} / \mathrm{mL}$, Fig. 4f, Supplementary Table 11). One RTG derived from 304 OS1364 had a lower production of ethyl acetate (Fig. 4f, Supplementary Figure 9c, Supplementary Table 305 11). This is consistent with recombination encompassing the gene $A T F 2$, which has a role in shaping this trait 306 (Supplementary Table 8). Moreover, the RTG derived from OS1364 showed diversified production of 307 esters, which may lead to a further differentiation of the sensory profile of the beer (Fig. 4f).

308 Overall, our data demonstrate that RTG recombination in sterile polyploid strains can unlock phenotypic 309 improvement in traits of industrial relevance ${ }^{16}$, contributing toward microbial stability or shaping the sensory 310 quality of the product. 
bioRxiv preprint doi: https://doi.org/10.1101/2021.10.21.465299; this version posted October $22,2021$. The copyright holder for this preprint (which was not certified by peer review) is the author/funder, who has granted bioRxiv a license to display the preprint in perpetuity. It is made available under aCC-BY-NC-ND 4.0 International license.

a

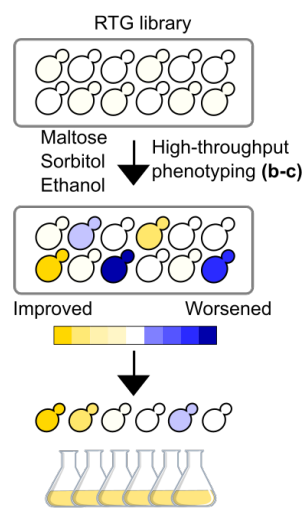

Flask scale high-gravity fermentation ( $32^{\circ}$ Plato) (d)

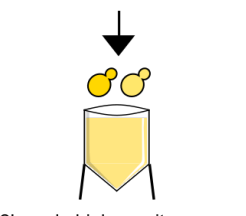

$2 \mathrm{~L}$ scale high-gravity fermentation (20 $20^{\circ}$ Plato) (e-f)

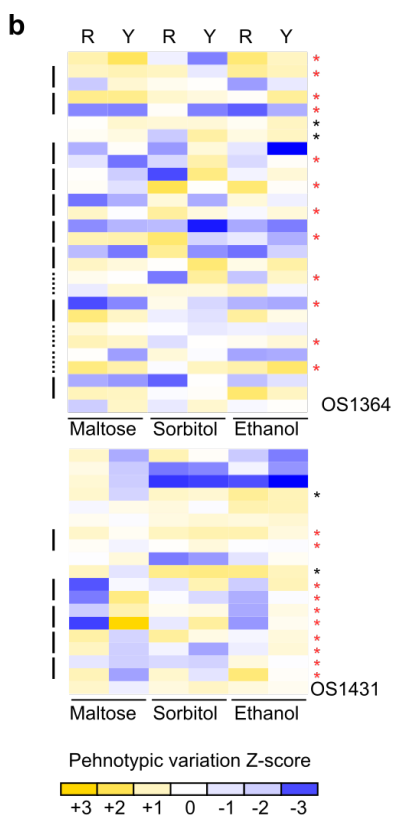

C

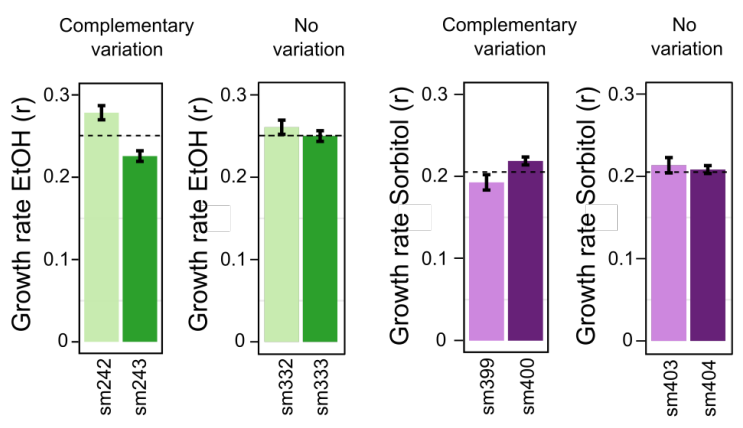

\begin{tabular}{|c|c|c|c|c|}
\hline & \multicolumn{2}{|c|}{$\begin{array}{c}\text { Complementary } \\
\text { variation M-D }\end{array}$} & \multicolumn{2}{|c|}{ No variation $M-D$} \\
\hline & OS1364 & OS1431 & OS1364 & OS1431 \\
\hline Maltose & 6 & 0 & 6 & 5 \\
\hline Sorbitol & 4 & 3 & 8 & 2 \\
\hline Ethanol & 7 & 1 & 5 & 4 \\
\hline
\end{tabular}

d
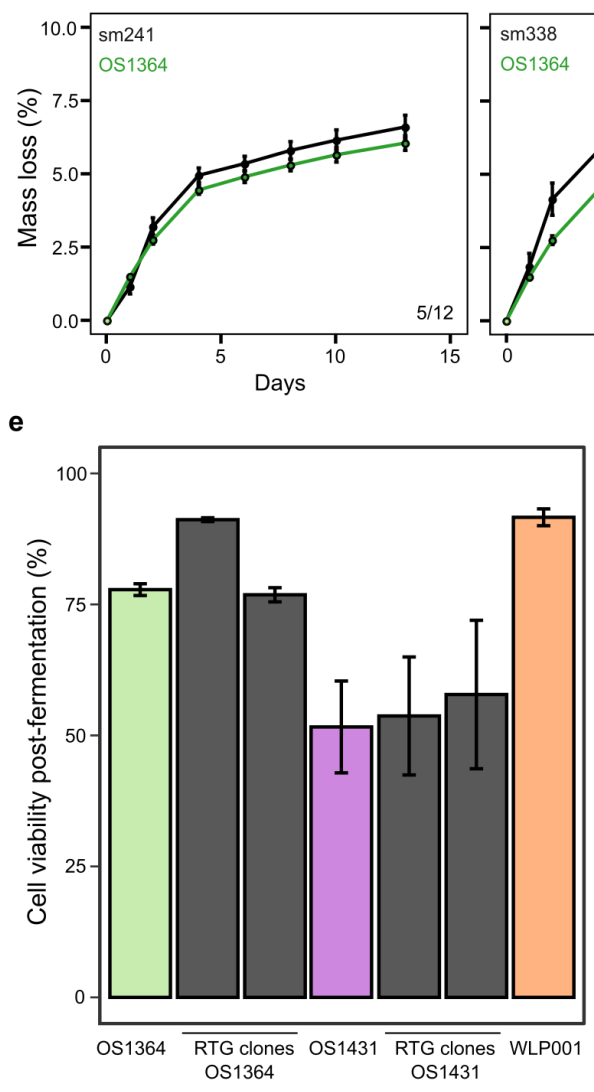

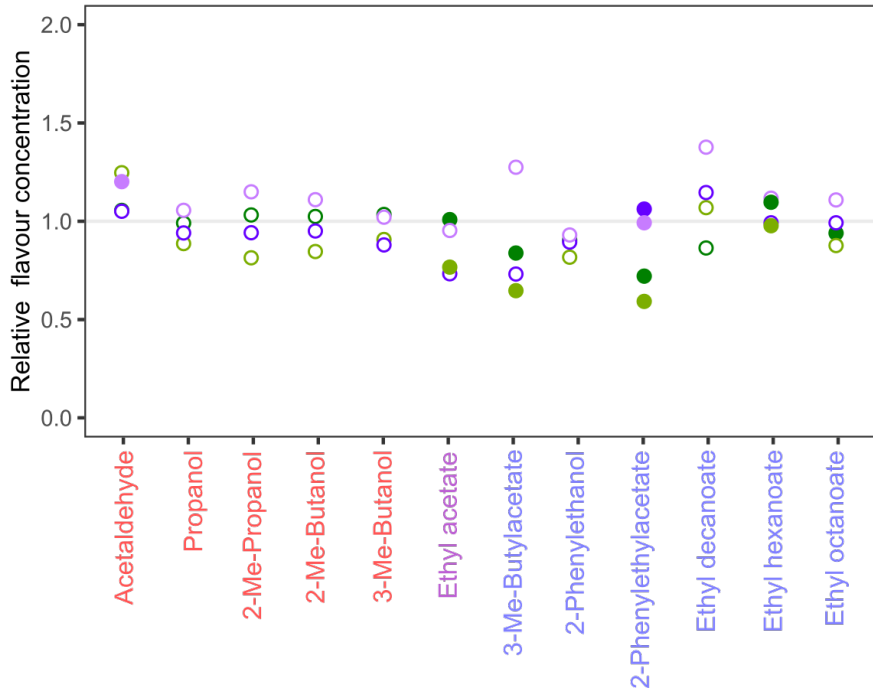

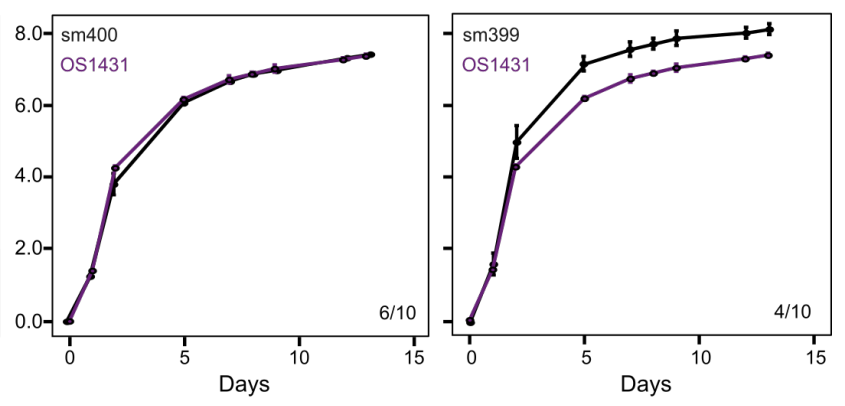

ays

sm244

sm338

- sm399

sm408

Filled: Above threshold Empty: Below threshold

\section{Unpleasant}

Unpleasant at high concentrations

Pleasant

Fig. 4. Phenotypic variability in industrial RTG variants | (a) Schematic of the phenotypic screening performed. Letters in parentheses indicate the respective data for the phenotypic screening depicted (b) Heatmaps representing growth rate $\left(1^{\text {st }}\right.$ column, $\left.\mathrm{R}\right)$ and maximal optical density $\left(2^{\text {nd }}\right.$ column, $\left.\mathrm{Y}\right)$ of the RTG samples selected for the next screening (red star), T0 samples (black star) and the M-D sectors (connected with lateral black line) and RTG-sectors for which only one sample of the sector (dotted-black line). Each measure is the average of 4 technical replicates. (c) Phenotypic diversification in sectored M-D pairs of OS1364 (green) and OS1431 (purple). Bar plots represent an example of an RTG M-D pair with divergent phenotypic variation and absence of variation. A similar example is 
reported on the left for OS1431. The dotted line represents the average phenotype in the RTG M-D library and the error bar represents the standard deviation. On the bottom table is reported the number of M-D pairs showing a complementary phenotypic variation per each class. (d) Mass loss curves during the flask scale fermentation experiment of an RTG without variation (left) and an improved RTG (right) for OS1364 (green) and OS1431 (purple), the error bars represent the standard deviation. The numbers on the bottom right represent the RTG samples in each category on the overall number of RTGs screened. (e) Post fermentation viability measured after a 2L-fermentation in high gravity wort for the two parents, the respective RTG samples and a commercial strain in triplicate. One RTG (sm244) derived from OS1364 showed a huge increased in viability. The error bars represent the standard deviation. (f) Variability in the aroma profile across the four RTGs from the $2 \mathrm{~L}$ scale fermentation, the values are expressed as relative change compared to the concentration produced by the parental strain.

\section{Discussion}

In this work, we showed that RTG is an efficient approach to generate genetic and phenotypic diversity in two fundamentally different industrial sterile yeast strains. Current approaches to improve industrial yeasts largely depend on designed targeted genetic modifications. Such approaches must contend with market restrictions and can garner societal mistrust ${ }^{16}$. As an alternative, we now show that RTG-assisted homologous recombination is an efficient approach to generate genetic and phenotypic diversity in polyploid and sterile industrial yeasts. The RTG approach, coupled with selection based on natural phenotypes generates non-GMO yeast strains that can be unrestrictedly introduced into the market. Compared to other non-GMO approaches such as serial transfer ${ }^{28,29}$, RTG induces a random genome shuffling as it does not select for a specific trait except for the genetic loci regulating the natural phenotype selected. Nevertheless, RTG has several benefits over the previous improvement strategies. We showed that the RTG process does not trigger genome instability in polyploid strains, similarly to diploid hybrids ${ }^{17,18}$. This is in contrast with direct selection where the abundant number of generations performed often results in ploidy and chromosome copy number variation $^{30}$. Furthermore, trade-offs in unselected traits have been described as undesirable outcomes in adaptive evolution experiments ${ }^{31-33}$, although some solutions have been proposed to fix, for instance, the impaired sugar consumption emerging after adaptive evolution ${ }^{34}$. We also observed RTG variants with impaired performances. In fact, we found that the RTG M-D pairs often had complementary worsened and improved fitness in different environments. In addition, multiple deleterious trade-offs can accumulate in RTG samples when the fraction of the genome that recombines is large. However, the RTG library harbours samples with variabile levels of genome-wide recombination and samples with a low level of recombination might be less likely to experience undesired trade-offs. Nevertheless, one of the most recombined RTG did not show phenotypic decay in the screenings performed; on the contrary, it was better able to tolerate the harsh fermentation conditions and had increased post-fermentative viability.

The RTG libraries are produced without introducing any selective pressure. Therefore, they are phenotypically agnostic, except for the trait used for the selection and can harbour improved phenotypes that are useful for multiple applications. The variability of "colony-associated-phenotypes" is a key parameter of the approach we devised for selecting M-D RTG recombinants, and it might be limited by the variability of natural phenotypes that can be screened upon RTG induction. However, a simultaneous screening of several environments could provide an effective solution to unveil variability in colony phenotypes. For instance, 
there are media which can trigger a colour variation linked to relevant industrial traits, such as the production of hydrogen sulfide detected in BiGGY agar plates ${ }^{35}$. Moreover, our approach successfully captured mother and daughter RTG pairs that have only been isolated so far with a micromanipulation approach ${ }^{18}$ developed for laboratory strains that cannot be scaled-up. We envision that our selection method can be easily automated to quickly screen hundreds of colonies ${ }^{36}$. In addition, our selection protocol can be easily performed and seamlessly integrated with other RTG methods that do not feature mother-daughter RTG pairs ${ }^{37}$ or other yeast improvement strategies. For instance, strains derived from an adaptive evolution experiment could be further improved through RTG to erase trade-offs, or, vice-versa, RTG samples could be submitted to adaptive evolution to be further optimized. In addition, the RTG framework can be easily generalized to reshuffle designed hybrid genomes ${ }^{17,18}$ as the only requirement is that the hybrid can enter meiosis and progress until the first meiotic prophase, even if with a low efficiency. The RTG approach has other practical applications as RTG libraries can be used in large-scale linkage analyses to unravel the genetic architecture of complex industrial traits and the RTG induced recombination could aid existing genome phasing methods ${ }^{38}$ in producing polyploid de-novo genome-assemblies. In conclusion, we propose the RTG framework as a novel avenue to induce genetic and phenotypic variability in industrial sterile yeasts. RTG can generate variants that can easily reach the market and can also serve as a method to help our understanding of complex genetic traits in industrial strains.

\section{Methods}

\section{Detection of deleterious, missense mutations and pre-existing LOHs}

381 Short reads of the parental strains were obtained from the 1011 S. cerevisiae yeast project ${ }^{13}$ and mapped against the SGD reference R64.2.1 using bwa-mem algorithm. Single nucleotide variants were called by using Freebayes (v1.3.1-19) with the argument "-p" to set an appropriate ploidy and quality $>20$. We annotated impactful mutations by using the Variant Effect Predictor (VEP) suite ${ }^{39}$. Mutations were annotated as impactful if they caused a stop-loss, stop-gain or start-loss. Subtelomeric variants or frameshift ones were included in the list to make the data comparable to the table of LOFs generated for the $1011 \mathrm{~S}$. cerevisiae yeast project ${ }^{13}$. The list of essential genes was obtained from Liu et $\mathrm{al}^{23}$. The GO-term analysis was performed using the SGD GO-term analysis suite at https://www.yeastgenome.org/ with a $p$-value threshold of 0.01 and selecting GO term associated with sporulation. We identified pre-existing LOHs in the genome of the parental strains by searching for non-overlapping regions of $50 \mathrm{kbp}$ where we identified 10 or less heterozygous markers that where not shared by all the unphased haplotypes. The plots were generated using ggplot2 and inhouse R scripts.

\section{Genome content analysis}

395 The genome content of OS1364 and OS1431 was measured using propidium iodide staining as described in Mozzachiodi et $\mathrm{al}^{17}$ and compared to a reference diploid strain. Each strain was patched from a $-80^{\circ} \mathrm{C}$ glycerol stock onto a YPD plate (1\% yeast extract, $2 \%$ peptone, $2 \%$ dextrose, $2 \%$ agar) and incubated overnight at 30 
${ }^{\circ} \mathrm{C}$. The following days the strains were incubated in $1 \mathrm{~mL}$ liquid YPD medium (1\% yeast extract, $2 \%$ peptone, $2 \%$ dextrose) and growth overnight at $30^{\circ} \mathrm{C}$ without shaking. The next day $200 \mu \mathrm{L}$ of the overnight culture was resuspended in $1 \mathrm{~mL}$ of fresh YPD and growth until exponential phase. Then cells were centrifugated, washed with $1 \mathrm{~mL}$ water and fixed overnight in $1 \mathrm{~mL}$ of EtOH 70\%. The following day each sample was washed with PBS 1X, resuspended in the PI staining solution ( $15 \mu \mathrm{M}$ PI, $100 \mu \mathrm{g} / \mathrm{mL}$ RNase A, $0.1 \% \mathrm{v} / \mathrm{v}$ Triton-X, in PBS) and incubated for 3 hours at $37^{\circ} \mathrm{C}$ in the dark. Ten thousand cells for each sample were analysed on a FACS-Calibur flow cytometer. Cells were excited at $488 \mathrm{~nm}$ and fluorescence was collected with a FL2-A filter. An increase of $50 \%$ of the fluorescence value (a.u.) compared to the fluorescence signal of the diploid cells in G1 was used to assign a ploidy of $3 \mathrm{n}$. An increase of $100 \%$ of the fluorescence value (a.u.) compared to the fluorescence signal of the diploid cells was used to assign a ploidy of $4 n$.

\section{Long read sequencing and HGT identification}

411 Yeast cells were grown overnight in liquid YPD medium. Genomic DNA was extracted using Qiagen 412 Genomic-Tips 100/G according to the manufacturer's instructions. The MINION sequencing library was 413 prepared using the SQK-LSK108 sequencing kit according to the manufacturer's protocol. The library was 414 loaded onto a FLO-MIN106 flow cell and sequencing was run for 72 hours. We performed long read 415 basecalling and scaffolding using the pipeline LRSDAY ${ }^{40}$. The Canu assembler mostly merged the different 416 haplotypes and thus prevented to produce long-read phased genomes. The dotplots were generated by using 417 mummerplot ${ }^{41}$. The annotated non-reference regions on chromosome XIII of OS1364 were extracted from the 418 fasta file of the collapsed assembly genomes (the haplotypes were not phased) and blasted by using the application "blastn" at https://www.ncbi.nlm.nih.gov/ against the database of ascomycetes.

\section{Sporulation monitoring and spore viability}

422 Yeast strains were patched on YPD plates and incubated at $30{ }^{\circ} \mathrm{C}$ for 24 hours. From the patch a streak for single was done and incubated at $30{ }^{\circ} \mathrm{C}$ for 48 hours, then single colonies were isolated and grown overnight at $30{ }^{\circ} \mathrm{C}$ in $10 \mathrm{~mL}$ of liquid YPD in a shaking incubator at $220 \mathrm{rpm}$. The following day, single colonies were inoculated in different tubes containing $10 \mathrm{~mL}$ of the pre-sporulation medium SPS (1\% peptone, $1 \%$ potassium acetate, $0.5 \%$ yeast extract, $0.17 \%$ yeast nitrogen base, $0.5 \%$ ammonium sulfate, $1.02 \%$ potassium biphthalate) and kept at $30^{\circ} \mathrm{C}$ in a shaking incubator for 24 hours. Tubes were then centrifuged and washed three times with sterile water and cells were resuspended in erlenmeyer flasks containing $25 \mathrm{~mL}$ of KAc $2 \%$ to reach a final $\mathrm{OD}$ of 1 , and incubated at $23^{\circ} \mathrm{C}$ in a shaking incubator. Sporulation was monitored by DAPI staining of the sporulation cultures to detect cells which have passed the first meiotic division, and contain two nuclei, or the second meiotic division, and contain four nuclei. The cells were first washed with water and then fixed with EtOH $70 \%$ overnight. Following, the cells were stained with DAPI and $10 \mu \mathrm{L}$ of stained cells were spread on a microscope slide and incubated in the dark for 40 minutes. To infer the efficiency of meiotic progression the cells were analysed by fluorescence microscopy and scored as having one, two or four nuclei, 
which indicates if they have not progressed after MI (one nucleus) or have progressed after MI (two nuclei) or MII (four nuclei). For assesing the spore viability, the spores were collected from the sporulated cultures and incubated between 30-60 minutes in $100 \mu \mathrm{L}$ of zymolase solution in order to perform spore dissection. At least 400 spores per sample were dissected on YPD plates. Spore viability was assessed as the number of spores forming visible colonies after 4 days of incubation at $30^{\circ} \mathrm{C}$.

\section{Plasmid engineering and genome editing with CRISPR/Cas9}

442 The multi-deletions of URA3, NDT80 and SPO11 were engineered by using CRISPR/Cas9 genome editing. 443 The plasmid harbouring Cas9 was obtained from Addgene pUDP004 ${ }^{42}$ and linearized with BsaI. The resistance to acetamide was replaced with the resistance cassette to Kanamycin. The gRNA with the necessary nucleotide for self-cleavage was designed on $\mathrm{UGENE}^{43}$ and ordered as a synthetic oligo from Eurofins Genomics $\left({ }^{\mathrm{TM}}\right)$. The synthetic oligo was cloned into the plasmid backbone by using the Gibson assembly kit (NEB, Gibson Assembly ${ }^{\circledR}$ ) and the ligation reaction was carried out for 1 hour at $50{ }^{\circ} \mathrm{C}$. The assembled plasmid was transformed into DH5-alpha competent bacteria by heat shock and the bacteria were incubated in reaction (PCR) using primers to validate the correct golden gate assembly of the construct. Successfully transformed bacterial colonies were inoculated in LB broth containing $100 \mu \mathrm{g} / \mathrm{uL}$ of ampicillin and incubated overnight at $37^{\circ} \mathrm{C}$. Cells were harvested from the overnight incubation and the plasmid was extracted using the QIAprep Spin Miniprep Kit following the manufacturer's instructions.

The 120 bp cassettes used for the deletion of URA3, NDT80 and SPO11 were designed to be flanking $60 \mathrm{bp}$ upstream and downstream the candidate gene which harbours the cutting site for Cas9 and ordered as a unique synthetic oligo at Eurofins Genomics. The forward and reverse cassettes were mixed at equimolar ratio, heated at $95{ }^{\circ} \mathrm{C}$ for 15 minutes and then cooled down at room temperature to be ready for the transformation. Yeast samples were transformed using between 1-15 ug of cassette, at least $200 \mathrm{ng}$ of CRISPR/Cas9 plasmid and following the protocol from ${ }^{42}$. Cells were then plated on selective media containing kanamycin (400 $\mathrm{ug} / \mathrm{mL}$ ) and incubated at $30^{\circ} \mathrm{C}$ for 3 - 7 days. Candidate transformed clones were validated by PCR using primers designed on the outside regions of the deleted genes. Positive clones were streaked for single on YPD and grown for 2 days at $30^{\circ} \mathrm{C}$ to allow plasmid loss. Plasmid loss was confirmed by plating again the colonies in the selective medium and positive ones were patched on YPD and stored at $-80{ }^{\circ} \mathrm{C}$ in $25 \%$ glycerol tubes.

\section{RTG selection by $U R A 3$-loss assay}

467 A URA3 cassette was introduced in one LYS2 locus on chromosome II in URA3 knock-out OS1364 and OS1431 strains by using the classical lithium acetate protocol. The correct insertion was validated by checking for restored prototrophy on synthetic media lacking uracil and by PCR. Positive clones were stored at $-80{ }^{\circ} \mathrm{C}$ in $25 \%$ glycerol tubes. For performing the URA3-loss assay, cells from the frozen stocks were 
and incubated at $30{ }^{\circ} \mathrm{C}$ for 2 days. Single colonies were picked and sporulation was induced following the protocol described in the paragraph "Sporulation monitoring and spore viability". Cells from the sporulation cultures were taken at 0,6 and 14 hours after sporulation induction, washed three times with YPD and incubated in YPD for 12 hours at $30^{\circ} \mathrm{C}$ without shaking. Dilutions of the YPD liquid culture were spotted onto YPD plates and an appropriate dilution was chosen for each strain and plated on 5-FOA (2\% dextrose, $0.675 \%$ yeast nitrogen base, $0.088 \%$ uracil drop-out, $0.005 \%$ uracil, $2 \%$ agar, $0.1 \% 5$-FOA plates. The plates were incubated at $30^{\circ} \mathrm{C}$ for 2 days and colonies growing on 5-FOA plates were counted at all the time points. We calculated the LOH rate at the three time points (T0, T6 and T14) as the ratio between the \% of cells growing on 5-FOA and the respective percentage of cells growing on YPD, according to the following equations:

$\mathrm{LOH} \mathrm{rate}_{\mathrm{T} 0}=100 \times\left(\mathrm{CFU}_{5-\mathrm{FOA}, \mathrm{T} 0} / \mathrm{CFU}_{\mathrm{YPD}, \mathrm{T} 0}\right)$

$\mathrm{LOH} \mathrm{rate}_{\mathrm{T} 6}=100 \mathrm{x}\left(\mathrm{CFU}_{5-\mathrm{FOA}, \mathrm{T} 6} / \mathrm{CFU}_{\mathrm{YPD}, \mathrm{T} 6}\right)$

$\mathrm{LOH}$ rate $_{\mathrm{T} 14}=100 \times\left(\mathrm{CFU}_{5-\mathrm{FOA}, \mathrm{T} 14} / \mathrm{CFU}_{\mathrm{YPD}, \mathrm{T} 14}\right)$

where $\mathrm{CFU}_{5-\mathrm{FOA}, \mathrm{TO}}$ is the number of colony forming units on 5-FOA at $\mathrm{T} 0$ and $\mathrm{CFU}_{\mathrm{YPD}, \mathrm{TO}}$ is the number of colony forming units on YPD at T0. These LOH rates were used to calculate: 1) the fold-increase of cells experiencing $\mathrm{LOH}$ by dividing the $\mathrm{LOH}$ rate at $\mathrm{T} 6$ or $\mathrm{T} 14$ by the $\mathrm{LOH}$ rate at $\mathrm{T} 0$ (LOH ratio); 2) the absolute difference of $\mathrm{LOH}$ by subtracting the $\mathrm{LOH}$ rate at $\mathrm{T} 0$ to the respective $\mathrm{LOH}$ rates at $\mathrm{T} 6$ and $\mathrm{T} 14$.

\section{RTG selection by natural phenotypes}

493 Sporulation of wild-type hybrids was induced as described in "Sporulation monitoring and spore viability" for 494 a time window compatible with RTG (14 hours). Cells were withdrawn from the sporulation medium before the appearance of MI cells to avoid plating committed meiotic cells. At the appropriate time point, cells were shifted from sporulation medium to liquid YPD. Part of the cells were incubated between $2-3$ hours to induce RTG but not budding and separation of the mother-daughter RTG pairs, while others were incubated longer to allow complete budding and separation of candidates M-D RTG pairs. Then, the samples were plated on modified YPD media (YPD $0.5: 1 \%$ yeast extract, 2\% peptone, $0.5 \%$ dextrose. YPD $1: 1 \%$ yeast extract, 2\% peptone, $1 \%$ dextrose) and plates were incubated at $30{ }^{\circ} \mathrm{C}$ and monitored daily for colony formation and morphology variation. Mother-daughter RTG pairs were selected as single colonies displaying two sectors with different morphology, of which one resembled the wild-type phenotype whereas the other was divergent. Cells from each side of the sectors were taken with a wooden stick and streaked on their respective modified YPD to limit contamination from cells of the other sector, and incubated at $30^{\circ} \mathrm{C}$ for 2 days. Then, a single colony was taken, patched on YPD and incubated at $30{ }^{\circ} \mathrm{C}$ for 2 days and finally stored in $25 \%$ glycerol tubes at $-80{ }^{\circ} \mathrm{C}$. Pictures of the sectoring colonies on the plates were taken with a stereomicroscope Discovery v. 8 


\section{CNVs detection in parental strains and RTG samples}

Short reads Illumina sequencings of were performed at the genomic platform of the Institute Curie. Reads were mapped to the S288C reference genome with the bwa-mem algorithm. Optical duplicates of the sequencing were removed using "samtools rmdup". Processed BAM files were then indexed using "samtools

\section{3} index" and coverage was extracted using "samtools depth". Coverage along the chromosomes was plotted using in-house R scripts in which sliding windows of non-overlapping $10 \mathrm{kbp}$ were used to calculate a local average coverage, which was then normalized with the median coverage along the chromosome. Genomewide coverage profiles were manually inspected to detect artifacts due to "smiley pattern" or lower coverage affecting only small chromosomes. Aneuploidies and large CNVs were detected by manually inspecting the $\log _{2}$ coverage profiles, while shorter CNVs $(<1 \mathrm{Kbp})$ were detected by computing the median coverage across sliding windows of non-overlapping $1 \mathrm{Kbp}$. Regions showing a coverage variation with respect to the median of their respective chromosome were then matched with the previously detected CNVs.

\section{LOH detection in RTG samples}

523 To identify LOHs in the evolved RTGs, we first generated a list of reliable markers represented by all the 524 heterozygous positions between the reference S288C strain and the two polyploid parental genomes (OS1364 525 and OS1431). Reads mapping and post-processing of sequenced parental strains, T0 and RTG samples were 526 performed as described in the previous paragraph "CNVs detection in parental strains and RTG 527 samples".Variant calling in the parental strains was performed by using Freebayes (v1.3.1-19) with the 528 options "-p" to set the appropriate ploidy. The parental vcf files were then filtered to include only "SNP" markers with quality $>20$ and depth $>10$ using bcftools with the options "TYPE=snp, QUAL $>20$, DP $>10$ ". 530 Variant calling on the evolved RTG and control (T0) clones was done using Freebayes with the options "-p" 531 to set the appropriate ploidy, “-@”" to call variants at the previously identified heterozygous positions and the 532 additional options "-m 30, -q 20 -i -X -u" for minimum depth, quality and to exclude complex variants. Vcfs were then filtered with bcftools to take only "SNP" variants. As an additional filtering step, the parental and samples vcf files were intersected using bedtools "-intersect" and variants with shared positions were extracted from the vcf of the RTG evolved samples. Next, we used an in-house R script to compare the frequencies of the alternative and reference alleles of each marker in the evolved samples and in the parental ones. We identified putative recombined regions as the ones showing an allele frequency shift in the markers, independently of its direction. Heterozygous markers present in the parental strain but not in the RTG samples were considered to have reached a fixed reference homozygous genotype if the position was covered during sequencing. Moreover, the allele of the marker tagged as being in an LOH region was compared with the known allele of the marker present in the ancestral strain and those not matching the expected allele were filtered out. Given the complexity of polyploids analysis, we decided to use a stringent threshold and we annotated regions as $\mathrm{LOH}$ only if they contained at least 9 consecutive recombined markers regardless of the directionality of the allele that is gain or lost due to recombination. In this way, we avoided calling false positive LOHs at the cost of decreasing our resolution. Among those LOHs, the ones overlapping for $>80 \%$ of 
their length among $>=70 \%$ of the samples considering each datasets separately (wild-type RTG, LYS2/URA3 selected RTG and $n d t 80 \triangle$ RTG) were filtered out. Additionally, LOH overlapping for $>=90 \%$ of their length with the ones found in the T0 sampleswere also excluded. LOHs spanning the LYS2/URA3 locus on chromosome II were not excluded but that chromosome was not counted for calculating the percentage of markers lying in $\mathrm{LOH}$ regions. Moreover, LOHs already present in the parental samples engineered with the LYS2/URA3 system, or in which NDT80 was deleted, were removed from the respective derived samples. LOHs in subtelomeric regions were excluded due to the unreliability of mapping in these highly repetitive regions. Finally, the list of annotated CNVs in core parts of the genome of the parental strains was used to filter the $\mathrm{LOH}$ detected. The plots of the $\mathrm{LOH}$ distribution were done using an in-house $\mathrm{R}$ script implemented with ggplot2 (v3.6.1) that takes into account the genotype shift of each marker in the LOH blocks.

\section{Inferring the parental haplotypes from the mother-daughter RTG pairs}

One M-D RTG pair derived from OS1364 was used as a proof of concept to phase a region on the left arm of chromosome IX where recombination likely resulted from a cross-over between two homologs. The genotype of each heterozygous marker was inferred based on the allele frequency (AF) shift in the M-D pair. For example, when we detected an AF shift toward the alternative allele in a marker, that is, the RTG sample has gained one alternative allele, the genotype of the allele was assigned as the reference for that marker. We followed the same approach when we detected an $\mathrm{AF}$ shift toward the reference allele and assigned in this case the "alternative" genotype. Then, the information of these recombined haplotypes was used to infer the third haplotype based on the allele dosage, which is the number of "Ref" or "Alt" copies estimated for each heterozygous marker. For instance, if the initial genotype was "Ref/Alt/Alt" and the AF shift detected was toward the reference allele, the third unknown haplotype harboured an "Alt" allele and the two recombining haplotypes had respectively a "Reference" and "Alternative" allele. The information of the recombined haplotypes were validated on a second RTG M-D pair having a recombination event spanning the same region.

\section{Microplate cultivations}

573 The osmotic stress and ethanol tolerance were assessed with microcultures in media containing $25 \%(\mathrm{w} / \mathrm{v}$ ) sorbitol and $8 \%(\mathrm{v} / \mathrm{v})$ ethanol, respectively. The microcultures were carried out in 100-well honeycomb microtiter plates at $25{ }^{\circ} \mathrm{C}$ (with continuous shaking), and their growth dynamics were monitored with a Bioscreen C MBR incubator and plate reader (Oy Growth Curves Ab, Finland). The wells of the microtiter plates were filled with $300 \mu \mathrm{L}$ of YPM medium (1\% yeast extract, $2 \%$ peptone, $1 \%$ maltose) supplemented with sorbitol $(25 \%)$ and ethanol $(8 \% \mathrm{v} / \mathrm{v})$. Control cultivations in media without sorbitol or ethanol were also carried out. Precultures of the strains were started in $20 \mathrm{~mL}$ YPM medium and incubated at $25{ }^{\circ} \mathrm{C}$ with shaking at $120 \mathrm{rpm}$ overnight. We measured the optical density at $600 \mathrm{~nm}$, and pre-cultures were diluted to a

581 final $\mathrm{OD}_{600}$ value of 3 . The microcultures were started by inoculating the microtiter plates with $10 \mu \mathrm{L}$ of cell 582 suspension per well (for an initial $\mathrm{OD}_{600}$ value of 0.1 ) and placing the plates in the Bioscreen $\mathrm{C}$ MBR. The 
optical density of the microcultures at $600 \mathrm{~nm}$ was automatically read every $30 \mathrm{~min}$. Four replicates were performed for each strain in each medium. Growth curves for the microcultures were modelled based on the $\mathrm{OD}_{600}$ values over time using the 'GrowthCurver'-package for $\mathrm{R}^{44}$.

\section{Flask-scale very high gravity wort fermentations}

$50 \mathrm{~mL}$-scale fermentations were carried out in $100 \mathrm{~mL}$ Schott bottles capped with glycerol-filled airlocks. Yeast strains were grown overnight in $25 \mathrm{~mL}$ YPM medium at $25{ }^{\circ} \mathrm{C}$. The pre-cultured yeast was then inoculated into $50 \mathrm{~mL}$ of $32^{\circ} \mathrm{P}$ wort made from malt extract (Senson Oy, Finland) at a rate of $7.5 \mathrm{~g}$ fresh yeast $\mathrm{L}^{-1}$. Fermentations were carried out in duplicate at $20^{\circ} \mathrm{C}$ for 13 days. Fermentations were monitored by mass lost as $\mathrm{CO}_{2}$. The alcohol content of the final beer was measured with an Anton Paar density meter DMA 5000 $\mathrm{M}$ with Alcolyzer beer ME and pH ME modules (Anton Paar GmbH, Austria).

\section{2-L scale high-gravity wort fermentations}

Strains were characterized in fermentations performed in a $20^{\circ} \mathrm{P}$ high gravity wort at $20^{\circ} \mathrm{C}$. Cultures were propagated essentially as described previously ${ }^{45}$, with the use of a "generation 0 " fermentation prior to the actual experimental fermentations. The experimental fermentations were carried out in triplicate, in 3-liter cylindroconical stainless steel fermenting vessels, containing 2 liters of wort medium. The $20^{\circ} \mathrm{P}$ wort $(98 \mathrm{~g}$ of maltose, $34.7 \mathrm{~g}$ of maltotriose, $24 \mathrm{~g}$ of glucose, and $6.1 \mathrm{~g}$ of fructose per liter) was produced at the VTT Pilot Brewery from barley malt and malt extract (Senson Oy, Finland). Fermentations were inoculated at a rate of 5 $\mathrm{g}$ fresh yeast $\mathrm{L}^{-1}$ (corresponding to approximately $15 \times 10^{6}$ viable cells $\cdot \mathrm{mL}^{-1}$ ). The wort was oxygenated to $10 \mathrm{mg} \cdot \mathrm{L}^{-1}$ prior to pitching (oxygen indicator model 26073 and sensor 21158; Orbisphere Laboratories, Switzerland). The fermentations were carried out at $20^{\circ} \mathrm{C}$ until the alcohol level stabilized, or for a maximum of 15 days. Wort samples were drawn regularly from the fermentation vessels aseptically and placed directly on ice, after which the yeast was separated from the fermenting wort by centrifugation $(9,000 \times \mathrm{g}, 10 \mathrm{~min}, 1$ ${ }^{\circ} \mathrm{C}$ ). Samples for yeast-derived flavour compound analysis were drawn from the beer when fermentations were ended. Cells viability was measured by propidium iodide staining of the cells that were collected at the end of the fermentations using a Nucleocounter ${ }^{\circledR}$ YC- $100^{\mathrm{TM}}$ (ChemoMetec, Denmark).

\section{Chemical analysis of fermentable sugars}

The concentrations of fermentable sugars (maltose and maltotriose) were measured by HPLC using a Waters 2695 separation module and Waters system interphase module liquid chromatograph coupled with a Waters 2414 differential refractometer (Waters Co., Milford, MA, USA). A Rezex RFQ-Fast Acid $\mathrm{H}^{+}(8 \%) \mathrm{LC}$ column $\left(100 \times 7.8 \mathrm{~mm}\right.$; Phenomenex, USA) was equilibrated with $5 \mathrm{mM} \mathrm{H}_{2} \mathrm{SO}_{4}$ (Titrisol, Merck, Germany) in water at $80{ }^{\circ} \mathrm{C}$, and samples were eluted with $5 \mathrm{mM} \mathrm{H}_{2} \mathrm{SO}_{4}$ in water at a $0.8 \mathrm{~mL} \cdot \mathrm{min}^{-1}$ flow rate. The alcohol level $(\% \mathrm{vol} / \mathrm{vol})$ of samples was determined from the centrifuged and degassed fermentation samples using an Anton Paar density meter DMA $5000 \mathrm{M}$ with Alcolyzer beer ME and pH ME modules (Anton Paar $\mathrm{GmbH}$, Austria). 


\section{Chemical analysis of aroma compounds in fermented beers}

622 Yeast-derived higher alcohols and esters were determined by headspace gas chromatography with flame 623 ionization detector (HS-GC-FID) analysis. Four-milliliter samples were filtered ( $0.45 \mu \mathrm{m}$ pore size) and 624 incubated at $60{ }^{\circ} \mathrm{C}$ for $30 \mathrm{~min}$, and then $1 \mathrm{ml}$ of gas phase was injected (split mode, $225^{\circ} \mathrm{C}$, split flow of 30 $625 \mathrm{~mL} \cdot \min ^{-1}$ ) into a gas chromatograph equipped with an FID detector and headspace autosampler (Agilent 6267890 series; Palo Alto, CA, USA). Analytes were separated on a HP-5 capillary column (50 m by $320 \mu \mathrm{m}$ by $6271.05 \mu \mathrm{m}$ column; Agilent, USA). The carrier gas was helium (constant flow of $1.4 \mathrm{~mL} \cdot \min ^{-1}$ ). The 628 temperature program was $50{ }^{\circ} \mathrm{C}$ for $3 \mathrm{~min}, 10^{\circ} \mathrm{C} \cdot \min ^{-1}$ to $100{ }^{\circ} \mathrm{C}, 5{ }^{\circ} \mathrm{C} \cdot \min ^{-1}$ to $140{ }^{\circ} \mathrm{C}, 15^{\circ} \mathrm{C} \cdot \min ^{-1}$ to $629260{ }^{\circ} \mathrm{C}$, and then isothermal for $1 \mathrm{~min}$. Compounds were identified by comparison with authentic standards 630 and were quantified using standard curves. 1-Butanol was used as an internal standard.

\section{References}

634 1. Diamond, J. Evolution, consequences and future of plant and animal domestication. Nature 418, 700707 (2002).

2. Hickey, J. M., Chiurugwi, T., Mackay, I. \& Powell, W. Genomic prediction unifies animal and plant breeding programs to form platforms for biological discovery. Nat. Genet. 49, 1297-1303 (2017).

3. Sander, J. D. \& Joung, J. K. CRISPR-Cas systems for editing, regulating and targeting genomes. Nat. Biotechnol. 32, 347-355 (2014).

4. Duan, S.-F. et al. The origin and adaptive evolution of domesticated populations of yeast from Far East Asia. Nat. Commun. 9, 2690 (2018).

5. Gallone, B. et al. Domestication and Divergence of Saccharomyces cerevisiae Beer Yeasts. Cell 166, 1397-1410.e16 (2016).

6. Steensels, J., Gallone, B., Voordeckers, K. \& Verstrepen, K. J. Domestication of Industrial Microbes. Curr. Biol. 29, R381-R393 (2019).

7. Gallone, B. et al. Interspecific hybridization facilitates niche adaptation in beer yeast. Nat. Ecol. Evol.

8. Krogerus, K., Magalhães, F., Vidgren, V. \& Gibson, B. Novel brewing yeast hybrids: creation and application. Appl. Microbiol. Biotechnol. 101, 65-78 (2017).

Gibson, B. et al. New yeasts-new brews: modern approaches to brewing yeast design and development. FEMS Yeast Res. 17, (2017).

10. García-Ríos, E. et al. Improving the Cryotolerance of Wine Yeast by Interspecific Hybridization in the Genus Saccharomyces. Front. Microbiol. 9, 3232 (2019).

11. De Chiara, M. et al. Domestication reprogrammed the budding yeast life cycle. (Evolutionary Biology, 2020).

12. Ono, J., Greig, D. \& Boynton, P. J. Defining and Disrupting Species Boundaries in Saccharomyces. Annu. Rev. Microbiol. 74, 477-495 (2020).

13. Peter, J. et al. Genome evolution across 1,011 Saccharomyces cerevisiae isolates. Nature 556, 339-344 
660 14. Bigey, F. et al. Evidence for Two Main Domestication Trajectories in Saccharomyces cerevisiae Linked to Distinct Bread-Making Processes. Curr. Biol. 31, 722-732.e5 (2021).

15. Loidl, J. Meiotic chromosome pairing in triploid and tetraploid Saccharomyces cerevisiae. Genetics 139, 1511-1520 (1995).

16. Steensels, J. et al. Improving industrial yeast strains: Exploiting natural and artificial diversity. FEMS Microbiol. Rev. 38, 947-995 (2014).

17. Mozzachiodi, S. et al. Aborting meiosis allows recombination in sterile diploid hybrids. Nature Communications, In press.

18. Laureau, R. et al. Extensive Recombination of a Yeast Diploid Hybrid through Meiotic Reversion. PLOS Genet. 12, e1005781 (2016).

19. Friedlander, G. et al. [No title found]. Genome Biol. 7, R20 (2006).

20. Dayani, Y., Simchen, G. \& Lichten, M. Meiotic Recombination Intermediates Are Resolved with Minimal Crossover Formation during Return-to-Growth, an Analogue of the Mitotic Cell Cycle. PLoS Genet. 7, e1002083 (2011).

21. Yue, J. X. et al. Contrasting evolutionary genome dynamics between domesticated and wild yeasts. Nat. Genet. 49, 913-924 (2017).

22. Marsit, S., Leducq, J., Durand, É. \& Marchant, A. Evolutionary biology through the lens of budding yeast comparative genomics. Nature reviews Genetics. doi:10.1038/nrg.2017.49

23. Liu, G. et al. Gene Essentiality Is a Quantitative Property Linked to Cellular Evolvability. Cell 163, 1388-1399 (2015).

24. Charles, J. S., Hamilton, M. L. \& Petes, T. D. Meiotic Chromosome Segregation in Triploid Strains of

25. D'Angiolo, M. et al. A yeast living ancestor reveals the origin of genomic introgressions. Nature 587, 420-425 (2020).

26. Granek, J. A. \& Magwene, P. M. Environmental and Genetic Determinants of Colony Morphology in Yeast. PLoS Genet. 6, e1000823 (2010).

27. Voordeckers, K. et al. Identification of a complex genetic network underlying $\mathrm{S}$ accharomyces cerevisiae colony morphology. Mol. Microbiol. 86, 225-239 (2012).

28. Mans, R., Daran, J.-M. G. \& Pronk, J. T. Under pressure: evolutionary engineering of yeast strains for improved performance in fuels and chemicals production. Curr. Opin. Biotechnol. 50, 47-56 (2018).

29. Perli, T., Moonen, D. P. I., van den Broek, M., Pronk, J. T. \& Daran, J.-M. Adaptive Laboratory Evolution and Reverse Engineering of Single-Vitamin Prototrophies in Saccharomyces cerevisiae. Appl. Environ. Microbiol. 86, e00388-20, /aem/86/12/AEM.00388-20.atom (2020).

30. Gerstein, A. C., Chun, H.-J. E., Grant, A. \& Otto, S. P. Genomic Convergence toward Diploidy in Saccharomyces cerevisiae. PLoS Genet. 2, e145 (2006).

31. Caspeta, L. et al. Altered sterol composition renders yeast thermotolerant. Science. 346, 75-78 (2014).

32. Caspeta, L. \& Nielsen, J. Thermotolerant Yeast Strains Adapted by Laboratory Evolution Show TradeOff at Ancestral Temperatures and Preadaptation to Other Stresses. MBio 6, e00431-15 (2015).

33. Caspeta, L., Chen, Y. \& Nielsen, J. Thermotolerant yeasts selected by adaptive evolution express heat stress response at $30^{\circ}$ C. Sci. Rep. 6, 27003 (2016).

34. Kuyper, M. et al. Evolutionary engineering of mixed-sugar utilization by a xylose-fermenting strain. 
35. Linderholm, A. L., Findleton, C. L., Kumar, G., Hong, Y. \& Bisson, L. F. Identification of Genes Affecting Hydrogen Sulfide Formation in Saccharomyces cerevisiae. Appl. Environ. Microbiol. 74, 1418-1427 (2008).

36. Ruusuvuori, P. et al. Quantitative analysis of colony morphology in yeast. Biotechniques 56, (2014).

37. Serero, A. et al. Recombination in a sterile polyploid hybrid yeast upon meiotic Return-To-Growth. Microbiol. Res. 250, 126789 (2021).

38. Abou Saada, O., Tsouris, A., Eberlein, C., Friedrich, A. \& Schacherer, J. nPhase: an accurate and contiguous phasing method for polyploids. Genome Biol 22, 126 (2021).

39. McLaren, W. et al. The Ensembl Variant Effect Predictor. Genome Biol. 17, 122 (2016).

40. Yue, J.-X. \& Liti, G. Long-read sequencing data analysis for yeasts. Nat. Protoc. 13, 1213-1231 (2018).

41. Kurtz, S. et al. Versatile and open software for comparing large genomes. Genome Biol. 5, R12 (2004).

42. Vries, A. R. G. De, Groot, P. A. De, Broek, M. Van Den \& Daran, J. M. G. CRISPR - Cas9 mediated gene deletions in lager yeast Saccharomyces pastorianus. Microb. Cell Fact. 1-18 (2017).

43. Okonechnikov, K., Golosova, O. \& Fursov, M. Unipro UGENE: a unified bioinformatics toolkit. Bioinformatics 28, 1166-1167 (2012).

44. Sprouffske, K. \& Wagner, A. Growthcurver: an R package for obtaining interpretable metrics from microbial growth curves. BMC Bioinformatics 17, 172 (2016).

45. Krogerus, K. et al. Ploidy influences the functional attributes of de novo lager yeast hybrids. Appl. Microbiol. Biotechnol. 100, 7203-7222 (2016).

\section{Acknowledgements}

This work was supported by Agence Nationale de la Recherche (ANR-13-BSV6-0006-01, ANR-18-CE120004, ANR-15-IDEX-01), Fondation pour la Recherche Médicale (EQU202003010413), UCA AAP Start-up Deep tech, CEFIPRA, convention CIFRE 2016/0582 between Meiogenix and ANRT. We thank D'Angiolo M. and Adekunle D. for their critical reading of the manuscript.

\section{Author contributions}

S.M., A.N., G.L., conceived the project, S.M., K.K., A.N., B.G., G.L. designed the experiments, S.M., K.K. performed the experiments and analyzed the data, S.M. and G.L. wrote the paper with input from K.K., B.G. and A.N.

\section{Competing interests}

A.N. and G.L. have a patent application on "Yeast strains improvement method" using return-to-growth (US20150307868A1). 
bioRxiv preprint doi: https://doi.org/10.1101/2021.10.21.465299; this version posted October 22,2021 . The copyright holder for this preprint (which was not certified by peer review) is the author/funder, who has granted bioRxiv a license to display the preprint in perpetuity. It is made available under aCC-BY-NC-ND 4.0 International license.

\section{Data and materials availability}

742 The phenotype data are available within the supplementary tables. The short reads have been deposited on the

743 SRA archive https://www.ncbi.nlm.nih.gov/sra with project number PRJNA770168. 